\title{
Bohr Hamiltonian with a potential having spherical and deformed minima at the same depth
}

\author{
Petrică Buganu ${ }^{1, *}$, Radu Budaca $^{1,2}$, and Andreea-Ioana Budaca $^{1}$ \\ ${ }^{1}$ Department of Theoretical Physics, Horia Hulubei - National Institute for Physics and Nuclear Engineering, Reactorului 30, Magurele, \\ Romania, RO-077125, POB-MG6 \\ ${ }^{2}$ Academy of Romanian Scientists, Splaiul Independenei 54, RO-050094, Bucharest, Romania
}

\begin{abstract}
A solution for the Bohr-Mottelson Hamiltonian with an anharmonic oscillator potential of sixth order, obtained through a diagonalization in a basis of Bessel functions, is presented. The potential is considered to have simultaneously spherical and deformed minima of the same depth separated by a barrier (a local maximum). This particular choice is appropriate to describe the critical point of the nuclear phase transition from a spherical vibrator to an axial rotor. Up to a scale factor, which can be cancelled by a corresponding normalization, the energy spectra and the electromagnetic E2 transition probabilities depend only on a single free parameter related to the height of the barrier. Investigations of the numerical data revealed that the model represents a good tool to describe this critical point.
\end{abstract}

\section{Introduction}

The motivation for the study developed in [1] and discussed here started from the paper [2], where an infinite square well potential in the $\beta$ variable is proposed to describe the critical point of the nuclear shape phase transition from the spherical vibrator to the axial deformed rotor. On the other hand, it is well-known [2], that for this critical point the potential has two minima, a spherical and a deformed one, separated by a maximum. Using the infinite square well, basically the maximum is neglected and moreover, the potential is completely flat. This choice was proved to be more appropriate to describe the critical point of the shape phase transition from the spherical vibrator to the $\gamma$-unstable rotor [3], while here, as one will see, the introduction of the barrier plays an important role for the description of the critical point and of its corresponding shapes.

The field of the nuclear shape phase transitions became richer and richer over the years since the two critical point models, using an infinite square potential, were introduced. These are conventionally called E(5) [3] and $X(5)$ [2], respectively. Most of these studies have been already reviewed and can be found in the following works [4-12]. Here the discussion will be restricted to a special class of potentials, called sextic oscillator potentials $[13,14]$. The solution proposed in [1] and presented here is significantly different by the previous ones [15-21] being more general and recovering important properties which were unavailable before. For example, the introduction of the barrier and the same depth for the spherical and deformed minima are new results, which as one will see brings important information about this critical point.

\footnotetext{
*e-mail: buganu@theory.nipne.ro
}

More details can be found in the original paper [1], while here we try to attract very briefly the attention on these results and on the future perspectives for developments of the subject.

The plan of the work is the following. In the next section, we present the model, namely, the Bohr-Mottelson Hamiltonian [22, 23] with a sextic potential [24, 25]. In Sec. 3, a discussion on the numerical results is made insisting on the effects introduced by the barrier for the critical point, while in the last section, the main results of the study are highlighted.

\section{Presentation of the model}

The Bohr-Mottelson Hamiltonian [22, 23] is solved for the critical point of the nuclear shape phase transition from the spherical vibrator to the axial symmetric rotor by using a sextic oscillator potential of the form [1]

$$
v(\beta)=a \beta^{2}+b \beta^{4}+c \beta^{6},
$$

where $a, b$ and $c$ are free parameters. More precisely, the potential (1) is involved in the $\beta$ equation, which in this case is:

$$
\left[-\frac{1}{\beta^{4}} \frac{\partial}{\partial \beta} \beta^{4} \frac{\partial}{\partial \beta}+\frac{L(L+1)}{3 \beta^{2}}+v(\beta)\right] \Psi(\beta)=\epsilon^{\beta} \Psi(\beta) .
$$

Here, by $L$ and $\epsilon^{\beta}$ are denoted the total angular momentum and energy. For the energy, a scaling property can be used,

$$
\epsilon^{\beta}(a, b, c)=a^{1 / 2} e^{\beta}\left(1, b a^{-3 / 2}, c a^{-2}\right),
$$

which is useful when the energy is normalized to the energy of the first excited state of the ground band and that 


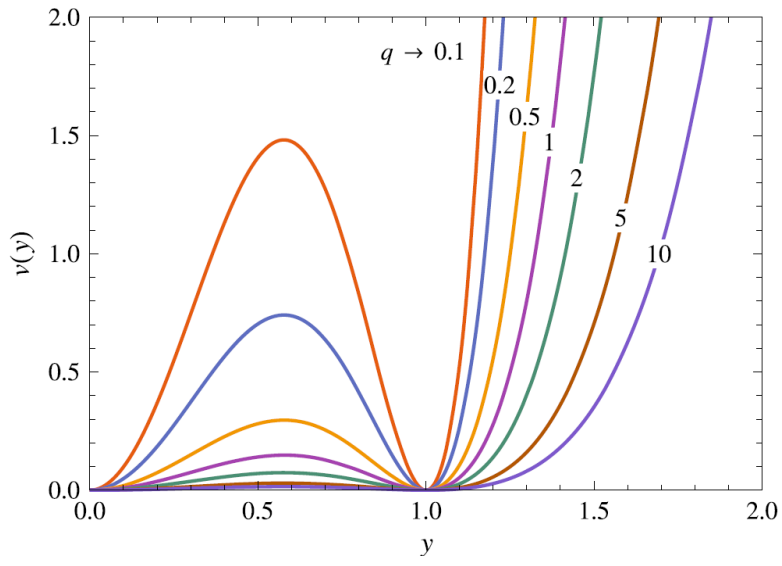

Figure 1. The potential (7) as a function of the $y$ variable for different values of $q$. The picture is taken from Ref. [1].

because in this case $a^{1 / 2}$ vanishes. For this scaling procedure, the potential (1) can be rewritten as:

$$
v(\beta)=\beta^{2}+\mu \beta^{4}+v \beta^{6} .
$$

According to Ref. [2], the potential for the critical point has to degenerated minima, a spherical and a deformed one. By imposing this condition for the potential (4), one gets

$$
v(\beta)=\beta^{2}-2 q \beta^{4}+q^{2} \beta^{6},
$$

where $q$ is a free parameter related to the height of the barrier. For numerical calculations it is useful to introduce the change of variable $y=\sqrt{q} \beta$, which transforms Eq. (2) in:

$$
\left[-\frac{\partial^{2}}{\partial y^{2}}-\frac{4}{y} \frac{\partial}{\partial y}+\frac{L(L+1)}{3 y^{2}}+v(y)\right] \Psi(y)=E \Psi(y),
$$

with the potential

$$
v(y)=\frac{1}{q^{2}}\left(y^{2}-2 y^{4}+y^{6}\right),
$$

and energy $E=e^{\beta} / q$. The potential (7) is plotted in Fig. 1 for different values of $q$. One can see that the barrier increases for $q \rightarrow 0$, while for large values of $q$ the barrier vanishes recovering the infinite square well limit.

Concerning Eq. (6), it is solved by expanding the wave function in an orthogonal basis [24, 25]:

$$
\Psi_{L k}(y)=\sum_{n}^{n_{\max }} A_{n}^{k} \tilde{\Psi}_{v n}(y),
$$

where

$$
v=\sqrt{\frac{9}{4}+\frac{L(L+1)}{3}},
$$

while $n_{\max } \rightarrow \infty$ and $k=n_{\beta}+1$. Here $\tilde{\Psi}_{v n}(y)$ are solutions of the same equation, but corresponding to an infinite square well potential:

$$
\tilde{v}(y)=\left\{\begin{array}{l}
0, \quad y \leq y_{W} \\
\infty, \quad y>y_{W}
\end{array}\right.
$$

The eigenvalues are obtained by diagonalizing the corresponding Hamiltonian matrix and truncating for an $n_{\max }$ which gives a very good convergence [1]:

$$
H_{n m}=\left(\frac{\alpha_{n}}{y_{W}}\right)^{2} \delta_{n m}+\frac{2 \sum_{i=1}^{3} v_{i}\left(y_{W}\right)^{2 i} I_{n m}^{(v, i)}}{q^{2} J_{v+1}\left(\alpha_{n}\right) J_{v+1}\left(\alpha_{m}\right)},
$$

where $v_{1}=v_{3}=1$ and $v_{2}=-2$. The computing of the integrals

$$
I_{n m}^{(v, i)}=\int_{0}^{1} x^{2 i+1} J_{v}\left(\alpha_{n} x\right) J_{v}\left(\alpha_{m} x\right) d x, x=y / y_{W},
$$

can be consistently expedited by employing some recurrence relations $[24,25]$. The boundary $y_{W}$ is fixed such that to obtain a certain minimal convergence of the diagonalization results for all considered states as its value is increased. The convergence radius $\epsilon=E\left(y_{W}^{i+1}\right)-E\left(y_{W}^{i}\right)$ for $y_{w}^{i+1}>y_{W}^{i}$ increases as one consider higher exited states. Therefore, the minimum precision, which in the present calculations is taken $\epsilon=10^{-7}$, is imposed on the highest exited state considered in numerical calculations. In this way, the energy for the lower states is automatically attained with a higher precision. The final boundary value $y_{W}$ encompass the relevant part of the original sextic potential, being considerably larger than the intersection point between the highest energy level and the sextic potential. As the total wave function is localized in one of the potential wells, before the outer wall of the potential, the boundary value once again covers it completely.

The model developed in [1] has been applied to analyze the shape of the nucleus as a function of the barrier in the critical point of the shape phase transition from the spherical vibrator to the axial rotor. Also, some numerical applications for the experimental data have been done. More details about these are shown in the next section.

\section{Numerical results and applications}

In this preliminary study [1], the energies are calculated only for the ground and $\beta$ bands, while those for the $\gamma$ band are part from a future work is being more difficult to calculate in the frame of the present model. These energies, normalized to the energy of the first excited state of the ground band, are plotted in Fig. 2 as a function of $\log _{10} q$. One can observe that for large values of $q$, the energy spectra correspond to the $\mathrm{X}(5)-\beta^{6}$ model [26], while for small values the limit of SU(3) is recovered. These numerical data can be used to find candidate nuclei for the model. A great curiosity, by starting this study, was to check the way in which the height of the barrier influences the shape of nucleus in the critical point. The introduction of the barrier, as can be seen from Fig. 3 and Fig. 4, changes dramatically the density probability distribution as a function of the deformation variable. For example, the density probability distribution for the ground state, in the case of the infinite square well potential, has a symmetric shape centered close to the middle of the potential, it has a large width, while, by introducing the barrier and increasing it, one can see from Fig. 4, that the peak becomes 


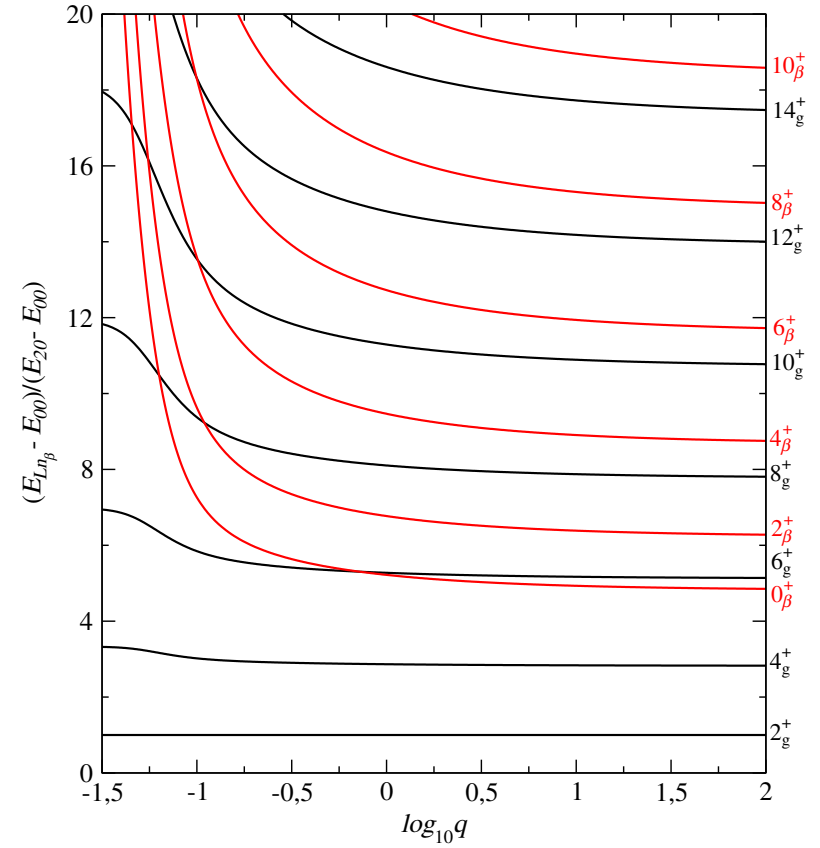

Figure 2. The energies, normalized to the energy of the first excited state of the ground band, plotted as a function of $\log _{10} q$. This picture is taken from [1].

very narrow and it is mostly centered around the deformed minimum. Also, the two peaks for the first state of the $\beta$ band are sensitive to the height of the barrier. The second peak decreases with the increase of the barrier, while the first peak prefers the spherical minimum. Moreover, from Fig. 4, panel (f), one can observe that three peaks show up.

One must remind here, that we are dealing with a radial-like equation, which in comparison to well studied one-dimensional double well potential does not contain the parity component which doubles up the spectrum of one of the wells when the barrier goes to infinity. It is then incorrect to consider the two wells of the sextic potential as separate when the barrier is very high. In the present case, the ground state rotational sequence is already localized in the deformed minimum. When the barrier is sufficiently high the excited rotational sequence becomes completely localized in the spherical minimum due to the quantum tunneling effect. At an infinite height of the barrier both rotational sequences recover the symmetrical rigid rotor spectrum $I(I+1) /(2 \mathcal{J})$, where $\mathcal{J}$ is the moment of inertia which is proportional with the square of the average deformation $\langle\beta\rangle^{2}$. Whereas the ground band states being localized in the deformed minimum have a finite non-zero deformation, the $\beta$ excited rotational band will have a mean deformation asymptotically going to zero as the barrier is further increased. Therefore, in the latter case, the $\beta$ excited energy band goes to infinity.

Taking into account the above results, on can conclude that the barrier brings new significant information at least regarding the energy spectra and the shape for this critical point, things which otherwise are unavailable. Thus, neglecting the barrier does not represent a good approximation, as has been thought so far.

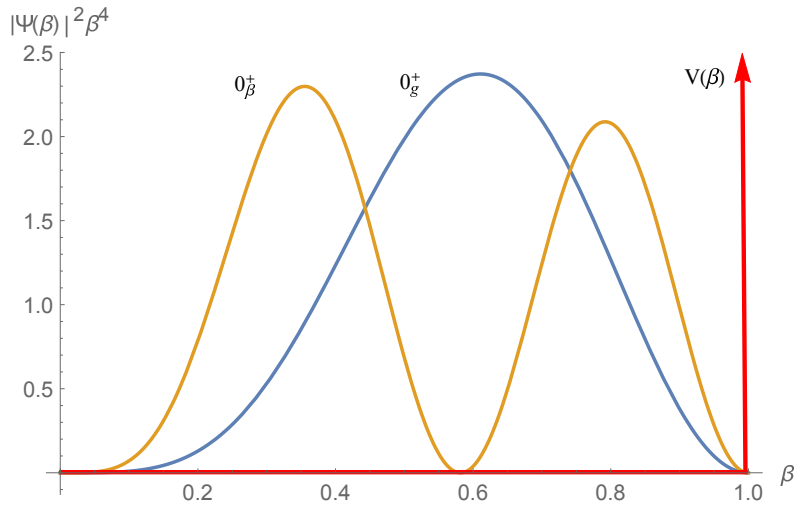

Figure 3. The density probability distribution as a function of the $\beta$ variable for the ground state $\left(0_{g}^{+}\right)$and for the first state of the $\beta$ band $\left(0_{\beta}^{+}\right)$for the infinite square well potential.

Because in Fig. 4, due to the height of the barrier, the density probability distribution has non-zero values for both minima, we asked ourself is a mixing or a coexistence of the two shapes show up here. We have looked in literature for other signatures related with this behavior and we have found [27] that usually strong monopole transition probability $\rho^{2}(E 0)$ could be connected with coexisting shapes having different deformations. Therefore, we have calculated [1] the monopole transition matrix element between the ground state and the head of the $\beta$ band and we have found large values for this when the barrier is high enough. The result is shown in Fig. 5.

Some numerical applications for experimental data have been made in [1]. Also, other two papers on this subject have been published in the meantime [28, 29] with new interesting results for the critical point of the nuclear shape phase transition from the spherical vibrator to the axial symmetric rotor. The diagonalization method discussed here can be easily extended for more complex polynomial potentials in the $\beta$ variable or to take into account other $\gamma$ axial deformations.

\section{Conclusions}

The Bohr-Mottelson Hamiltonian with a sextic potential is diagonalized in a basis of Bessel functions of the first kind. Using a scaling procedure, finally, the eigenvalues depend only on a free parameter related with the height of the barrier. The energy spectrum is sensitive to the height of the barrier, therefore, one can conclude that neglecting the barrier is not a really good approximation.

Analyzing the density distribution probability for the ground state and for the first excited state of the $\beta$ band, but also the monopole transition probability between these two states, one can see how the barrier influences the deformation of the ground and excited states and moreover, the fact that these states could present shape coexistence signatures.

From these preliminary results, one can conclude that the introduction of the barrier has to be taken into account for a better description of this critical point. Further stud- 

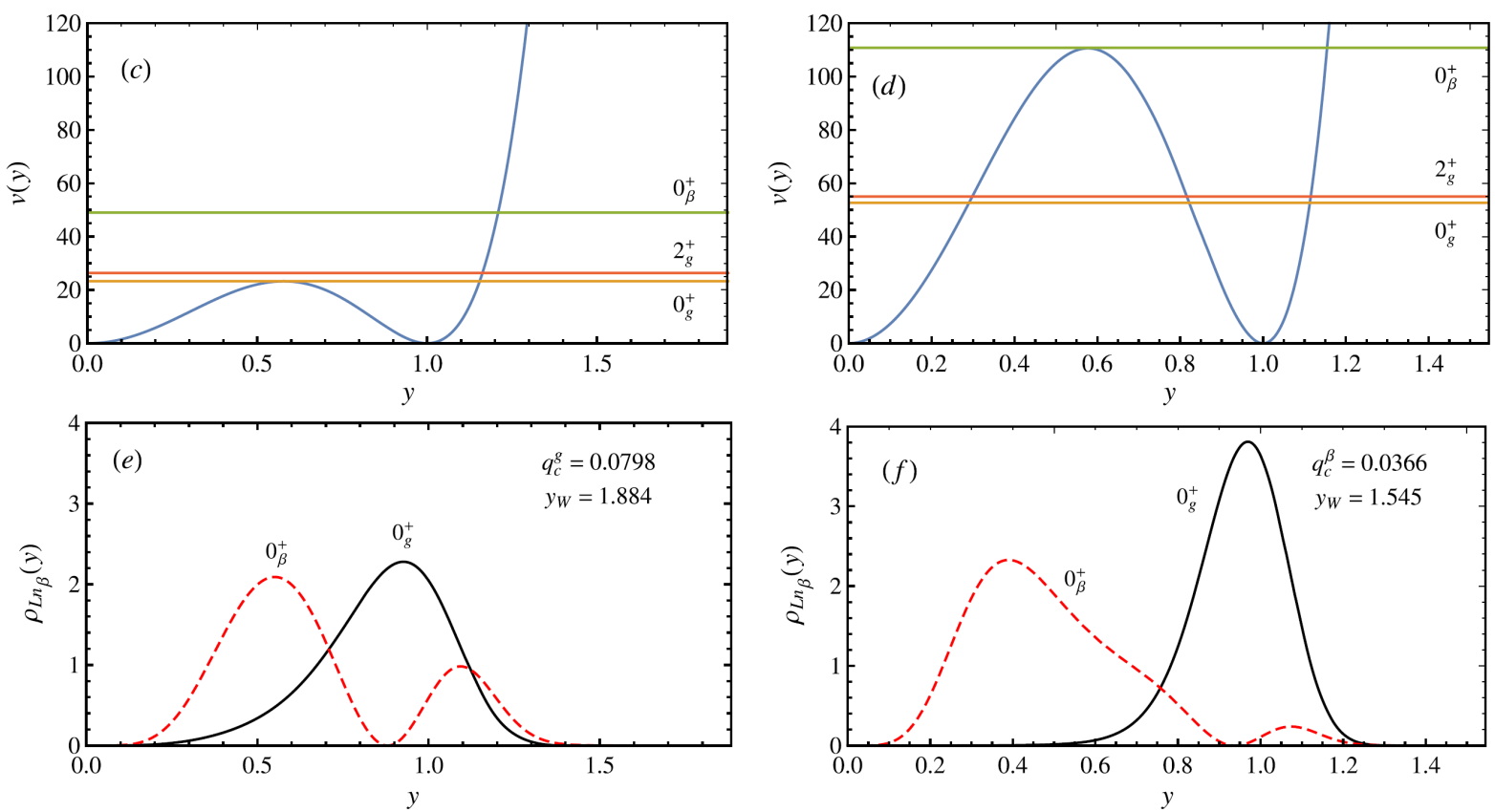

Figure 4. The potential and the absolute values for the $0_{g}^{+}, 2_{g}^{+}$and $0_{\beta}^{+}$energy levels are given in the same arbitrary units for the critical values $q_{c}^{g}$ (c) and $q_{c}^{\beta}$ (d). The critical values are pointed out in the corresponding contour plots with a white horizontal line. The probability distribution $y$-dependent profiles for the ground state and the $\beta$ band head state are shown in figures (e) and (f). This picture is taken from [1].

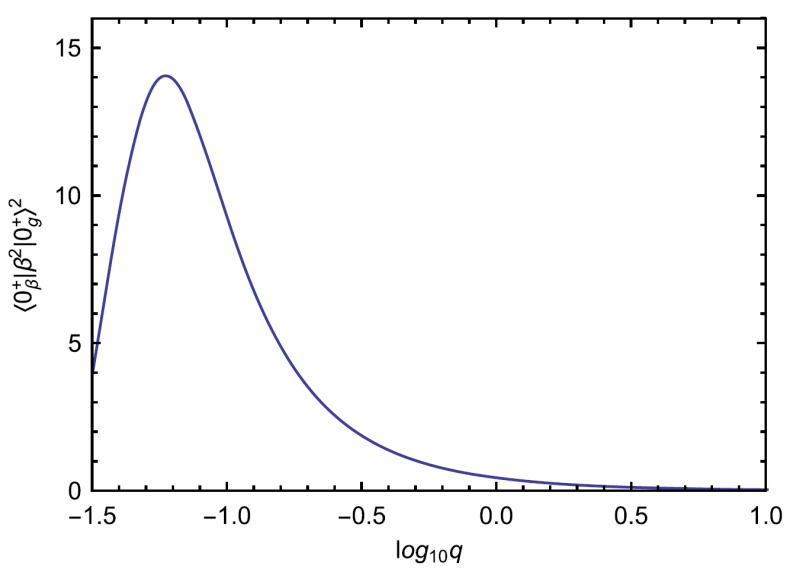

Figure 5. Monopole transition probability in units of $\left(\frac{3}{4 \pi}\right)^{2} Z^{2} \beta_{M}^{4}$ as a function of $\log _{10} q$. This picture is taken from [1].

ies, as relaxing the degeneracy between the spherical and deformed minima, introduction of the $\gamma$ band, adding octic or decadic terms to the potential are in preparation. Also, the model has to be applied for a large number of experimental data, in order to identify experimental realisation of this shape phase transition with a barrier in the critical point.

This work was supported by a grant of Ministry of Research and Innovation, CNCS - UEFISCDI, project No. PN-III-P1-1.1-TE2016-0268, within PNCDI III.

\section{References}

[1] R. Budaca, P. Buganu, A. Budaca, Phys. Lett. B 776, 26-31 (2018)

[2] F. Iachello, Phys. Rev. Lett. 87, 052502 (2001)

[3] F. Iachello, Phys. Rev. Lett. 85, 3580-3583 (2000)

[4] L. Fortunato, Eur. Phys. J. A 26, 1-30 (2005)

[5] R. F. Casten, Nature Phys. 2, 811-820 (2006)

[6] R. F. Casten, E. A. McCutchan, J. Phys. G: Nucl. Part. Phys. 34, R285 (2007).

[7] L. Prochniak, S. G. Rohozinsky, J. Phys. G: Nucl. Part. Phys. 36, 123101 (2009)

[8] D. J. Rowe, J. L. Wood, Fundamentals of Nuclear Models: Fundational Models, (Singapore: Wolrd Sicentific, 2010)

[9] P. Cejnar, J. Jolie, R. F. Casten, Rev. Mod. Phys. 82, 2155 (2010)

[10] A. Leviatan, Prog. Part. Nucl. Phys. 66, 93-143 (2011)

[11] P. Van Isacker, K. Heyde, Scholarpedia 9, 31279 (2014)

[12] P. Buganu, L. Fortunato, J. Phys. G: Nucl. Part. Phys. 43, 093003 (2016)

[13] R. Budaca, P. Buganu, M. Chabab, A. Lahbas, M. Oulne, Ann. Phys. (NY) 375, 65-90 (2016)

[14] P. Buganu, Proc. Ro. Acad. Ser. A 19, 455-462 (2018)

[15] G. Lévai, J. M. Arias, Phys. Rev. C 69, 014304 (2004)

[16] A. A. Raduta, P. Buganu, Phys. Rev. C 83, 034313 (2011) 
[17] A. A. Raduta, P. Buganu, J. Phys. G: Nucl. Part. Phys. 40, 025108 (2013)

[18] P. Buganu, R. Budaca, Phys. Rev. C 91, 014306 (2015)

[19] P. Buganu, R. Budaca, J. Phys. G: Nucl. Part. Phys. 42, 105106 (2015)

[20] R. Budaca, Eur. Phys. J. A 50, 87 (2014)

[21] R. Budaca, Phys. Lett. B 739, 56 (2014)

[22] A. Bohr, Mat. Fys. Medd. Dan. Vid. Selsk. 26, No. 14 (1952)

[23] A. Bohr, B. R. Mottelson, Mat. Fys. Medd. Dan. Vid. Selsk. 27, No. 16 (1953)
[24] H. Taseli, A. Zafer, Int. J. Quant. Chem. 63, 935 (1997)

[25] H. Taseli, A. Zafer, J. Comput. Appl. Math. 95, 83 (1998)

[26] D. Bonatsos, D. Lenis, N. Minkov, P. P. Raychev, P. A. Terziev, Phys. Rev. C 69, 014302 (2004)

[27] J. L. Wood, C. De Coster, K. Heyde, Nucl. Phys. A 651, 323-368 (1999)

[28] R. Budaca, P. Buganu, A. I. Budaca, Bulg. J. Phys. 44, 319-326 (2017)

[29] R. Budaca, A. I. Budaca, EPL 123, 42001 (2018) 